\title{
Alterations of ingestive behavior following trigeminal lemniscus lesions in rats
}

\author{
JAN E. WEISSENBURGER and VERNE C. COX \\ University of Texas at Arlington, Arlington, Texas 76019
}

\begin{abstract}
Previous research has suggested that inadvertent damage to the trigeminal lemniscus may account for some impairments of feeding behavior attributed to lateral hypothalamic lesions. In the present study, rostrally placed trigeminal lemniscus lesions produced no greater impairments in feeding or drinking behavior than observed in surgical control animals. Earlier reports of disturbed feeding following trigeminal lemniscus lesions may reflect damage to adjacent structures, particularly at caudal lesion sites.
\end{abstract}

One of the fiber systems recently implicated in the control of ingestive behavior is the sensory trigeminal lemniscus (Zeigler \& Karten, 1974). At the diencephalic level, the fibers of this lemniscus pass rostrally near the lateral hypothalamic (LH) nucleus proper (Smith, 1973). Zeigler and Karten have suggested that, due to their close proximity, some of the behavioral deficits typically attributed to damage of the $\mathrm{LH}$ area may actually reflect damage to the trigeminal fiber system. They have reported that some components of the LH syndrome (Teitelbaum \& Epstein, 1962) appear to result from damage restricted to the trigeminal sensory system. Specifically, trigeminal lesions in rats were found to be followed by a period of aphagia and adipsia similar to that previously reported to follow LH lesions. Zeigler and Karten's results are compatible with findings of Marshall and Teitelbaum (1974), indicating that sensory feedback from the muzzle area is related to feeding behavior. The latter researchers demonstrated that rats given LH lesions were not responsive to somesthetic stimulation of the muzzle area. Such a deficit may reflect incidental damage to the trigeminal fibers passing near the LH.

Extensive direct investigation of the trigeminal system has been conducted by Zeigler and Karten $(1973 a, 1973 b, 1974)$ in both pigeons and rats. The study of the trigeminal system in rats (Zeigler \& Karten, 1974) employed large asymmetric lesions covering much unspecified tissue and was based on data collected from a small number of animals (Stricker, Rowland, \& Zigmond, 1975).

In the present experiment, lesions of the trigeminal lemniscus were more restricted, and the number of lesioned animals employed was greater than in previous work. Postsurgery data were obtained for food intake, water intake, and body weight. The more rostral of two lesion sites used by Zeigler and Karten (1974)

This research was supported in part by the Organized Research Fund of the University of Texas at Arlington. was chosen for the lesion placement in the present experiment. This site was near the termination of the trigeminal lemniscal fibers in the ventrobasal thalamus, where the trigeminal fibers are fairly well isolated from other fiber systems, such as the medial forebrain bundle and pallidofugal and nigrostriatal pathways, which also pass near or through the $\mathrm{LH}$ area and have been implicated in LH syndrome deficits (Morgane, 1961; Ungerstedt, 1971). The more caudal sites used by Zeigler and Karten were considered less desirable because of the proximity of several sensory or motor fiber systems.

\section{METHOD}

\section{Subjects}

The subjects were 40 experimentally naive male albino rats (Holtzman Company), approximately 90 days of age, weighing $350-400 \mathrm{~g}$. All animals were individually housed in a temperature-controlled room and kept on a 12-h light-dark cycle. They were allowed ad-lib access to tap water and powdered Purina Lab Chow. After 1 week of adaptation to the laboratory, baseline measures of daily food intake were taken for all animals for 1 week. The animals were divided into three groups equalized on the basis of the baseline food intake data.

\section{Surgical Procedure}

One group of 20 animals received bilateral lesions of the trigeminal fiber system; a group of 10 animals received sham operations; and the remaining group of 10 served as unoperated controls. All operated animals were anesthetized with an intraperitoneal injection of Chloropent $(2.5 \mathrm{cc} / \mathrm{kg})$. For animals in the lesioned group, small, bilateral, electrolytic lesions were made in the trigeminal fiber system with a dc lesion maker utilizing a current intensity of $.5 \mathrm{~mA}$ for $10 \mathrm{sec}$. The stereotaxic coordinates were, with head level, $4.5 \mathrm{~mm}$ posterior to bregma, $1.2 \mathrm{~mm}$ lateral to the midline, and $7.3 \mathrm{~mm}$ below the skull surface. Previous pilot work established that these coordinates typically yield lesions of the trigeminal lemniscus at the level of 3,290 microns anterior to the frontal zero plane, according to the atlas of König and Klippel (1963). At this level, the trigeminal lemniscus is near its termination in the ventrobasal thalamus and is located near the dorsomedial edge of the zona incerta. Animals in the sham-operated group underwent identical surgical procedures, except that the electrodes were lowered only $6 \mathrm{~mm}$ below the skull, with no application of current. 


\section{Data Collection}

Following surgery, animals were returned to their individual cages and immediately allowed ad-lib access to powdered chow and water. Beginning the day after surgery, measures of food and water intake were taken once every $24 \mathrm{~h}$ for all animals. Postsurgical body-weight measures were begun on the eighth day following surgery.

Once the postlesion food intake of the lesioned and sham groups reached asymptotic levels, animals in these groups were tested for nonprandial drinking. Immediately following a 24-h water-deprivation period, each animal's food was removed and water consumption for the following $1 \mathrm{~h}$ was measured.

After the conclusion of testing, all animals were sacrificed with an overdose of Chloropent. Following intracardial perfusion with a $.9 \%$ saline solution followed by $10 \%$ formalin, brains of the lesioned and sham-operated animals were removed, frozen, and sectioned. Sixty-micron sections were stained with cresylecht violet and analyzed to determine the extent and placement of lesions and other tissue damage. Data from all lesioned animals with acceptable histology were included in subsequent analyses. The criterion for acceptable histology in these animals was defined as the presence of small, bilaterally symmetric lesions localized in the path of the trigeminal fiber tract.

\section{RESULTS}

Histological examination revealed that 9 of the 20 animals in the original lesioned group met criteria for acceptable neural damage. These animals had bilaterally symmetric damage generally extending no more than $1 \mathrm{~mm}$ in the anterior-posterior plane. Figure 1 illustrates the maximal extent of the lesion in a representative animal. Sham-operated animals displayed superficial damage from electrode passage, which was, in most cases, restricted to the top cortical layers of tissue.

Data from the nine histologically acceptable lesioned animals were used in all the subsequent statistical analyses. One sham animal died of surgical complications, leaving data from nine animals in this group for subsequent analyses. In order to simplify statistical analyses, data from one randomly selected control animal was eliminated to achieve an equal number of animals in each group.
Presurgery mean baseline food intake values, which were used as the basis for presurgical composition of lesion, sham, and control (intact) groups of animals were as follows: lesion, $26.65 \mathrm{~g}$; sham, $26.55 \mathrm{~g}$; and control, $26.60 \mathrm{~g}$. Postsurgery body-weight, food, and water intake data from all animals in all three groups were analyzed initially by mixed two-factor repeatedmeasures analyses of variance. The analysis for food intake resulted in significant days $[\mathrm{F}(18,36)=13.22$, $\mathrm{p}<.001]$ and Groups by Days interaction effects $[F(36,432)=2.49, p<.001]$. Analysis of food intake on the first day postsurgery resulted in a significant groups effect $[F(2,24)=132.72, p<.001]$. Orthogonal comparisons of the first postsurgical day's data revealed a significant difference between the two operated groups and the controls $[F(1,24)=265.36, p<.001]$ and no significant difference between sham and lesioned groups $[F(1,24)=.08, p>.05]$. The group means for daily food intake are shown in Figure 2. As can be seen, the postsurgery mean food intake for the sham and lesioned groups were initially much lower than corresponding values for the control group. The values for sham and lesioned groups increased over days to a level near that maintained by the control group.

A mixed two-factor repeated-measures analysis of variance for water intake resulted in significant days $[\mathrm{F}(18,36)=4.56, \mathrm{p}<.001]$ and Groups by Days interaction effects $[F(36,432)=1.86, p<.01]$. These results are similar to those found with food intake. Similarly, results of an analysis of water intake on the first day postsurgery resembled those for food intake, revealing a significant groups effect $[F(2,24)=13.64, p<.001]$. Orthogonal comparisons among means for water intake on the first postsurgical day yielded a significant difference between the two operated groups and the intact control group $[F(1,24)=27.19, p<.001]$, and no significant difference between sham and lesioned groups $[F(1,24)=.09, p>.05]$. As can be seen in Figure 3, the initial results for water intake were similar to those found for food intake in the immediate post-

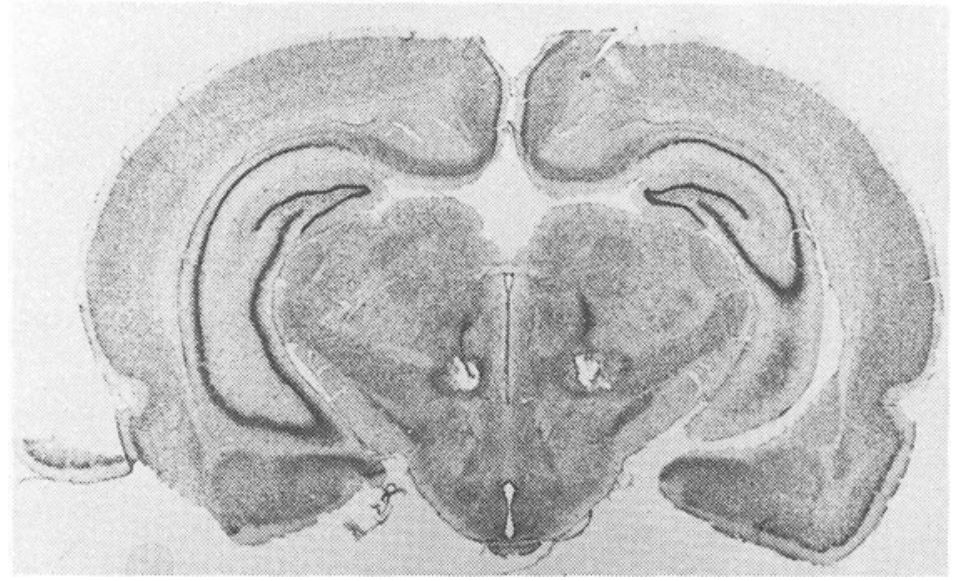

Figure 1. Representative coronal section from a histologically acceptable lesioned animal, showing maximal extent of the lesion. 


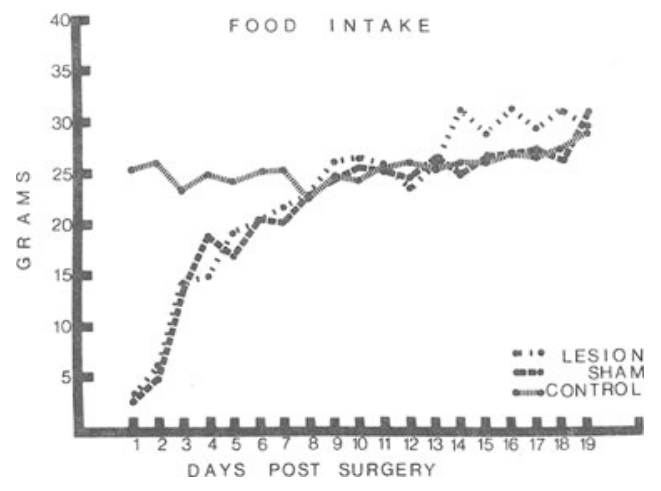

Figure 2. Mean values on food intake for lesioned, sham, and control groups for 19 days postsurgery.

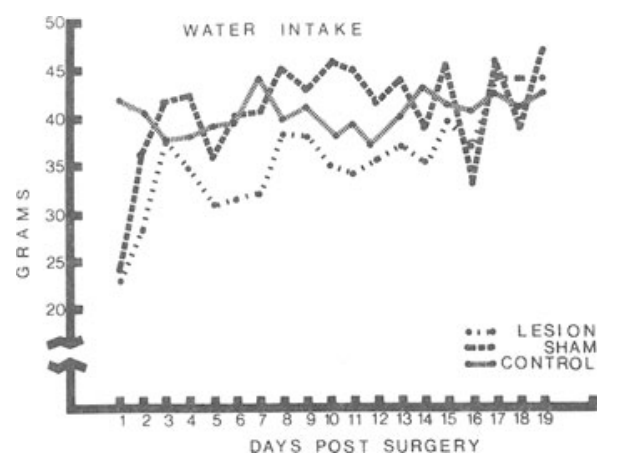

Figure 3. Mean values on water intake for lesioned, sham, and control groups for 19 days postsurgery.

surgery period, with sham and lesioned groups showing immediate postsurgery deficits of equivalent magnitude. Following Day 4, values for the lesioned group appeared lower for most of the remaining postsurgical period. However, an ANOVA limited to Days 5-19 failed to yield significant group $[\mathrm{F}(2,24)=.8, \mathrm{p}>.25]$, days $[F(14,336)=.53, p>.25]$, or interaction effects $[F(28,336)=1.07, p>.25]$. Body weight, beginning on Day 8 postsurgery, was analyzed by a mixed twofactor repeated-measures analysis of variance, which yielded no significant main or interaction effects $(p>.05)$. Both surgery groups showed normal bodyweight values by Day 8 postsurgery, despite the initial postsurgery decrease in food and water intake.

Inspection of individual food and water intake values for the first few days postsurgery reveals that four animals in the lesioned group were aphagic for 1 or 2 days (less than $1 \mathrm{~g} /$ day), and one was adipsic for 1 day (less than $1 \mathrm{ml} /$ day). Three animals in the sham group were aphagic for 1 or 2 days, but none were adipsic.

The nonprandial drinking test resulted in mean group water intake values for the 1-h sample of $2.75 \mathrm{ml}$ for the lesioned group, ${ }^{1} 3.44 \mathrm{ml}$ for the sham-operated group, and $5.9 \mathrm{ml}$ for the control group. The $\mathrm{F}$ ratio for the analysis fell between the .05 and .10 levels of significance $[F(2,23)=3.11, .10>p>.05]$. The results of a nonparametric test (Kruskal-Wallis) analysis were similar to the parametric test results, with the level of significance greater than $.05\left[\chi^{2}(2)=4.61\right.$, $.10>\mathrm{p}>.05]$.

\section{DISCUSSION}

Recent research regarding regulatory feeding and drinking systems has focused on the role of fiber systems that pass rostrally near, and sometimes terminate in, the LH nucleus (Stricker \& Zigmond, 1976). Each of these systems may make specific contributions to motivational states and regulatory behavior. The present study attempted to further explore the specific role of the trigeminal fiber system in food and water intake regulation. The results of the study indicate that bilateral damage to the trigeminal lemniscal fibers at a level near their termination in the ventrobasal thalamus does not yield aphagia and adipsia beyond that induced by sham surgery at a more dorsal site. The transient deficits in food and water intake found in the present study were manifest to an equivalent degree in sham-operated animals. Therefore, the deficits obtained appear related to surgical trauma rather than to neural damage specific to the trigeminal lemniscus. These results suggest that Zeigler and Karten's (1974) findings are due to damage to nontrigeminal structures.

A recent study by Neill and Kaufman (1977) examined feeding and drinking behavior after lesions in the ventrobasal thalamus in rats. This study is relevant to the present study, since the trigeminal lemniscal fibers branch out and terminate in this area. Aphagia was reported for 5 of 13 rats with thalamic lesions, and hypophagia was reported for the remaining lesioned animals and all control animals. However, there were no statistically significant differences in time to recovery of normal feeding in lesioned or control animals. Neill and Kaufman also reported feeding, drinking, and sodium appetite regulatory deficits following ventrobasal thalamic lesions. Of these regulatory functions, the only one directly tested for in the present study was water intake in the absence of food (nonprandial drinking). Although the data were not statistically significant in the present study, the trigeminal lemniscus lesioned animals consumed an average of $2.75 \mathrm{ml}$ in a 1-h period; intact controls consumed an average of $5.9 \mathrm{ml}$. Had a sample been taken for a 1.5-h period, as in the Neill and Kaufman study, differences in nonprandial drinking may have achieved statistical significance. Additional research will be required to determine if trigeminal lemniscus lesions yield food and sodium appetite deficits, as did ventrobasal thalamic lesions in the Neill and Kaufman study. Their study suggests that, unlike trigeminal lemniscus lesions, ventrobasal thalamic lesions may yield aphagia. Such a difference, as well as others between the two lesion sites, may reflect damage to additional systems at the ventrobasal thalamic site, such as the termination sites of gustatory fibers (Norgen \& Leonard, 1973).

\section{REFERENCES}

KönIG, J. F. R., \& KLIPPEL, R. The rat brain: A stereotaxic atlas of the brainstem. Baltimore: Williams \& Wilkens, 1963.

Marshall, J. F., \& Teitelbaum, P. Further analysis of sensory inattention following lateral hypothalamic damage in rats. Journal of Comparative and Physiological Psychology, 1974, 86, 375-395.

Morgane, P. J. Alterations in feeding and drinking behavior of rats with lesions in globi pallidi. American Journal of Physiology, 1961, 201, 420-428. 
Neill, D. B., \& Kaufman, J. L. Deficits in behavioral responding to regulatory challenges after lesions of ventrobasal thalamus in rats. Physiology \& Behavior, 1977, 19, 47-52.

Norgren, R., \& Leonard, C. M. Ascending central gustatory pathways. Journal of Comparative Neurology, 1973, 150, 217-238.

SмIтн, R. L. The ascending fiber projections from the principal trigeminal nucleus in the rat. Journal of Comparative Neurology, 1973, 148, 423-446.

Stric Ker, E. M., Rowland, N., \& Zigmond, M. J. Trigeminal lemniscal lesions and the lateral hypothalamic syndrome. Science, 1975, 190, 694-695.

Stricker, E. M., \& Zigmond, M. J. Recovery of function after damage to central catecholamine-containing neurons: A neurochemical model for the lateral hypothalamic syndrome. In J. M. Sprague \& A. M. Epstein (Eds.), Progress in psychobiology and physiological psychology (Vol. 6). New York: Academic Press, 1976.

Teitelbaum, P., \& Epstein, A. The lateral hypothalamic syndrome: Recovery of feeding and drinking after lateral hypothalamic lesions. Psychological Review, 1962, 69, 74-90.

UNGERSTEDT, U. Adipsia and aphagia after 6-OHDA induced degeneration of the nigrostriatal dopamine system. Acta Physiological Scandanavia, 1971, Suppl. 367, 95-122.

Zeigler, H. P., \& Karten, H. J. Brain mechanisms and feeding behavior in the pigeon (Columbia Livia) I. Quinto-frontal structures. Journal of Comparative Neurology, 1973, 152, 59-82. (a)

Zeigler, H. P., \& Karten, H. J. Brain mechanisms and feeding behavior in the pigeon (Columbia Livia) II. Analysis of feeding behavior deficits after lesions of quinto-frontal structures. Journal of Comparative Neurology, 1973, 152, 83-102. (b)

Zeigler, H. P., \& Karten, H. J. Central trigeminal structures and the lateral hypothalamic syndrome in the rat. Science, 1974. 186, 636-638.

\section{NOTE}

1. This value was computed on data from eight animals, due to spillage in one case.

(Received for publication August 7, 1978.) 